\title{
An Anisotropic Spatial Modeling Approach for Remote Sensing Image Rectification
}

Ke-Sheng Cheng, ${ }^{*},+$ Hui-Chung Yeh, ${ }^{*}$ and Chang-Hsuan Tsai

\begin{abstract}
$R$ 2ectification of a remote sensing image is commonly done by applying polynomial regression models to image coordinates and map coordinates of ground control points. A major drawback of the polynomial regression model is that it does not capture the random characteristic of terrain elevation. In fact, the distortion of a remote sensing image is attributed to the variation of terrain elevation and orbital parameters, the variations being random in nature. A more effective approach of remote sensing image rectification is a stochastic approach that takes into account the spatial variation structure of terrain elevation. This article presents an anisotropic spatial modeling approach of image rectification using ordinary kriging estimation. By considering the residuals of polynomial trend mapping as anisotropic random fields, the proposed approach models separately the spatial variation structures of the residuals in $\mathrm{X}$ and $\mathrm{Y}$ directions, and employs the ordinary kriging method for spatial interpolation of the residual random fields. By means of a cross validation procedure, residuals of image rectification by the polynomial trend mapping, the multiquadric interpolation function, and the ordinary kriging approaches are compared. The ordinary kriging approach yields smallest variances and root-mean-squared of mapping errors. CElsevier Science Inc., 2000
\end{abstract}

\section{INTRODUCTION}

Integration of remote sensing images and other geographic information for various applications has increased dra-

* Agricultural Engineering Department, National Taiwan University, Taiwan, Republic of China

$\uparrow$ Hydrotech Research Institute, National Taiwan University, Taiwan, Republic of China

Address correspondence to K.-S. Cheng, Agricultural Engineering Department and Hydrotech Research Inst., National Taiwan Univ., Taiwan, Republic of China. E-mail: rslab@ccms.ntu.edu.tw

Received 14 June 1999; revised 17 December 1999. matically over the last decade. No remote sensing images are free of geometric distortions and an essential requirement for integrated processing of remote sensing images and data from geographic information systems (GIS) is that they are spatially referenced. The geometric distortions inherent in remote sensing images fall into two categories, the systematic and nonsystematic distortions. Image rectification, a process by which the geometry of an image area is made planimetric (Haralick, 1973; Schowengerdt, 1997), must be performed prior to any subsequent analyses. Many factors, such as scan skew, mirror scan velocity, panoramic distortion, platform velocity, curvature of the earth, and earth rotation, contribute to the systematic distortions. This type of distortions can be rectified using data from platform ephemeris and knowledge of internal sensor distortion. Nonsystematic distortions arise from sensor system's attitude, velocity, and altitude, and can be corrected only through the use of ground control points (GCPs) (Jensen, 1986). In particular, topographic, or relief displacement due to terrain variation is usually the most serious of the displacement types, especially in mountainous terrain (Paine, 1981). There are essentially two different categories of image rectification techniques. One is the deterministic approach that establishes models for the nature and magnitude of the sources of distortion and uses these models to establish correction formulae. The deterministic approach relies on data of the flight parameters and the terrain information, and is effective when the types of distortion are well characterized, such as that caused by earth rotation (Richards, 1995). The other approach of image rectification is the statistical approach, which, by means of a GCP data set, establishes mathematical relationship between image coordinates and their corresponding map coordinates using standard statistical procedures. These relationships can be used to correct the image geometry irrespective of the analyst's knowledge of the source and type of distortion (Richards, 1995). The most widely used method in this category is the 
polynomial trend mapping (PTM) technique that employs polynomial regression equations to relate image coordinates and their corresponding map coordinates. The order of polynomials to be used depends on the image sources and the terrain variation in the scene. For airborne images or images from areas of rugged terrain, more severe distortions present and higher order polynomials are used. In contrast, affine transformation, a firstorder polynomial, may be sufficient to rectify satellite images. An extended model of the conventional polynomial mapping is the Hardy's multiquadric interpolation function (MIF), which yields zero errors for coordinate mapping at GCPs (Hardy, 1990; Star et al., 1997). The MIF method employs a distance-weighting algorithm to adjust residuals from the PTM method.

Work of recent research (Malinverno, 1988; Turcotte, 1988; Chilès, and Delfiner, 1999) has shown that terrain variation is random in nature and can be modeled as a random field. Therefore, distortions raised by terrain variation are randomly distributed over the spatial extent of an image. Neither the polynomial trend mapping nor the multiquadric interpolation function considers the spatial variation structure of the terrain elevation. In this study we propose an alternative approach of image rectification by kriging. Kriging is a group of spatial interpolation methods based on the spatial correlation structure of the random field under investigation. We adopted the ordinary kriging $(\mathrm{OK})$, a linear, unbiased estimator with minimum variance of estimation errors, for spatial interpolation in this research.

The organization of this paper is as follows. The next section introduces the PTM and MIF algorithms of image rectification. The third section describes theory of the ordinary kriging. The fourth section presents the algorithm of building a kriging model for image rectification. The fifth section describes the method of accuracy assessment by means of cross validation. An example is presented in the sixth section to illustrate the proposed model and compare the mapping accuracies by different approaches. The final section presents some concluding remarks.

\section{GEOMETRIC CORRECTION ALGORITHMS}

Since the statistical approach is commonly adopted for operational purpose and is independent of the platform used for data acquisition, we focus our study on modeling the geometric distortions based on ground control point correspondence. Ground control points are ground features that can be delineated on a remotely sensed image and on a corresponding map. Before proceeding, readers should be aware that geometric correction process described in this article is only the first phase of a complete remote sensing georeference process. It is followed by a resampling process, which interpolates digital numbers of pixels of the rectified image, using techniques like nearest neighbor, bilinear interpolation, and cubic convolution inter- polation. This study only deals with the geometric correction, and the process of pixel resampling is not part of our investigation. We introduce the PTM and MIF geometric correction algorithms in this section.

\section{Polynomial Trend Mapping}

Let $(x, y)$ be the image coordinate of a pixel and $(X, Y)$ be its corresponding map coordinate. Also let $(\hat{X}, \hat{Y})$ be estimate of the map coordinate $(X, Y)$ by a polynomial transformation. General form of the PTM algorithm is given in Eqs. (1a) and (1b):

$$
\begin{aligned}
& \hat{X}=\sum_{i=0}^{n} \sum_{j=0}^{i} a_{i j} x^{i-j} y^{j}, \\
& \hat{Y}=\sum_{i=0}^{n} \sum_{j=0}^{i} b_{i j} x^{i-j} y^{j} .
\end{aligned}
$$

Here $n$ is the order of the polynomial model, and the coefficients $a_{i j}$ and $b_{i j}$ are estimated by least-squares regression analysis. The PTM is basically a statistical trend surface mapping model. Using a set of $m$ GCPs, $\left[\left(x_{k}, y_{k}\right)\right.$; $\left.\left(X_{k}, Y_{k}\right)\right], k=1,2, \ldots, m$, it finds the best-fit surface for the GCP data set. As was introduced earlier, low order polynomials are used to rectify images with less geometric distortions, like satellite images from a flat region. Geometric distortions like scale, translation, rotation, and skew effects can be modeled by an affine transformation $(n=1)$. Rectification of images from rugged terrain may require using higher order polynomials. However, in choosing the order of polynomials for image rectification, one needs to be cautious that higher order polynomials can lead to significant image distortions for regions outside the range of the ground control points, although they generally yield accurate fits in the vicinities of the ground control points.

\section{Multiquadric Interpolation Function}

The MIF algorithm can be viewed as an extension of the PTM technique. The basic concept of this technique is to adjust $(\hat{X}, \hat{Y})$, the PTM estimate of map coordinate $(X, Y)$, by means of a distance-weighted algorithm. The weights are determined such that the MIF mapping errors at ground control points are zero.

The first step of the MIF technique is to apply the PTM model with lower degree ( 1 or 2 ) polynomials to the GCP data set, that is [Eqs. (2a) and (2b)],

$$
\begin{aligned}
& \hat{X}_{k}=a_{00}+a_{10} x_{k}+a_{11} y_{k}+a_{20} x_{k}^{2}+a_{21} x_{k} y_{k}+a_{22} y_{k}^{2}, \\
& \hat{Y}_{k}=b_{00}+b_{10} x_{k}+b_{11} y_{k}+b_{20} x_{k}^{2}+b_{21} x_{k} y_{k}+b_{22} y_{k}^{2} .
\end{aligned}
$$

Let residuals of the PTM model at ground control points, $\left(X_{k}, Y_{k}\right), k=1,2, \ldots, m$, be $\left(d X_{k}, d Y_{k}\right)$ [Eqs. (3a) and (3b)]:

$$
\begin{aligned}
& d X_{k}=X_{k}-\hat{X}_{k}, \\
& d Y_{k}=Y_{k}-\hat{Y}_{k} .
\end{aligned}
$$

The MIF algorithm then uses $\left\{\left(d X_{k}, d Y_{k}\right), k=1,2, \ldots\right.$, 
$m\}$ and the following steps to establish distance-weighted estimates for any non-GCP pixel $\left(x_{0}, y_{0}\right)$ (Star et al., 1997).

1. Calculate the distance between a non-GCP point $\left(x_{0}, y_{0}\right)$ and a $\operatorname{GCP}\left(x_{k}, y_{k}\right)$ [Eq. (4)]:

$$
f_{0 k}\left(x_{0}, y_{0}\right)=\sqrt{\left(x_{0}-x_{k}\right)^{2}+\left(y_{0}-y_{k}\right)^{2}} \text {. }
$$

2. Calculate the distance between two control points $\operatorname{GCP}\left(x_{k}, y_{k}\right)$ and $\operatorname{GCP}\left(x_{l}, y_{l}\right)$ [Eq. (5)]:

$$
f_{k l}=\sqrt{\left(x_{k}-x_{l}\right)^{2}+\left(y_{k}-y_{l}\right)^{2}} .
$$

3. Model the residuals $\left(d X_{k}, d Y_{k}\right), k=1,2, \ldots, m$, by the following matrix equations:

$$
\begin{aligned}
& {\left[\begin{array}{c}
d X_{1} \\
d X_{2} \\
\vdots \\
d X_{m}
\end{array}\right]=\left[\begin{array}{cccc}
f_{11} & f_{12} & \ldots & f_{1 m} \\
f_{21} & f_{22} & \ldots & f_{2 m} \\
\vdots & \vdots & \vdots & \vdots \\
f_{m 1} & f_{m 2} & \ldots & f_{m m}
\end{array}\right] \cdot\left[\begin{array}{c}
\alpha_{1} \\
a_{2} \\
\vdots \\
\alpha_{m}
\end{array}\right],} \\
& {\left[\begin{array}{c}
d Y_{1} \\
d Y_{2} \\
\vdots \\
d Y_{m}
\end{array}\right]=\left[\begin{array}{cccc}
f_{11} & f_{12} & \ldots & f_{1 m} \\
f_{21} & f_{22} & \ldots & f_{2 m} \\
\vdots & \vdots & \vdots & \vdots \\
f_{m 1} & f_{m 2} & \cdots & f_{m m}
\end{array}\right] \cdot\left[\begin{array}{c}
\beta_{1} \\
\beta_{2} \\
\vdots \\
\beta_{m}
\end{array}\right],}
\end{aligned}
$$

or, equivalently,

$$
D=\left[\begin{array}{l}
D_{X} \\
D_{Y}
\end{array}\right]=\Psi \cdot\left[\begin{array}{l}
A \\
B
\end{array}\right],
$$

where $D_{X}$ and $D_{Y}$ represent vectors of residuals in $X$ and $Y$ directions, $A$ and $B$ are column vectors of $\alpha_{k}$ and $\beta_{k}$, respectively, and $\Psi$ is the interGCP-distance matrix, that is [Eq. (6d)],

$$
\Psi=\left[\begin{array}{ll}
F & 0 \\
0 & F
\end{array}\right],
$$

with $F$ being the distance matrix in Eqs. (6a) and $(6 \mathrm{~b})$.

4. Solve Eq. (6c) for $\alpha_{k}$ and $\beta_{k}, k=1,2, \ldots, m$,

$$
\left[\begin{array}{l}
A \\
B
\end{array}\right]=\Psi^{-1} \cdot\left[\begin{array}{c}
D_{X} \\
D_{Y}
\end{array}\right]
$$

The solution is unique since there are $m$ equations for $m$ unknowns in Eqs. (6a) and (6b). Coefficients $\alpha_{k}$ and $\beta_{k}$ can be viewed as the residuals contributed by $\left(x_{k}, y_{k}\right)$ per unit length of distance from point $\left(x_{k}, y_{k}\right)$. From Eq. (7), we see that $\alpha_{k}$ and $\beta_{k}$ depend on the geometric layout of ground control points, which is characterized by $\Psi$, and the predetermined PTM residuals $D_{X}$ and $D_{Y}$. An implicit assumption of the MIF algorithm is that residuals contributed by $\left(x_{k}, y_{k}\right)$ are isotropic since the distance matrices $F$ and $\Psi$ are independent of directions.

5. Perform a geometric interpolation at $\left(x_{0}, y_{0}\right)$ by Eqs. (8a), (8b), and (9):

$$
d X_{0}=\sum_{k=1}^{m} f_{0 k} a_{k}
$$

$$
\begin{gathered}
d Y_{0}=\sum_{k=1}^{m} f_{0 k} \beta_{k}, \\
\left(\hat{X}_{M 0}, \hat{Y}_{M 0}\right)=\left(\hat{X}_{0}, \hat{Y}_{0}\right)+\left(d X_{0}, d Y_{0}\right) .
\end{gathered}
$$

Here, $\left(\hat{X}_{0}, \hat{Y}_{0}\right)$ and $\left(\hat{X}_{M 0}, \hat{Y}_{M 0}\right)$ are the PTM and MIF estimates of map coordinate $\left(X_{0}, Y_{0}\right)$, respectively. The MIF algorithm yields exact estimations, that is, zero estimation errors, at ground control points since Eq. (6c) has a unique solution. The residuals at $\left(x_{0}, y_{0}\right)$ are determined by summing the distance-weighted residuals, $f_{0 k} \alpha_{k}$ and $f_{0 k} \beta_{k}, k=1,2, \ldots, m$, contributed by all ground control points.

\section{THEORY OF THE ORDINARY KRIGING}

The PTM is basically a traditional trend surface mapping model. It finds the best-fit surface for the GCP data set. The MIF interpolates the residuals resulted from PTM by weighting residuals contributed by each ground control point. Neither method considers the spatial variation structure of terrain elevation. The terrain variation structure may be nonlinear and anisotropic. In contrast, kriging is a group of spatial estimation methods that take into account the spatial variation of the random field under investigation. In this study, we apply the most widely used ordinary kriging to remote sensing image rectification and introduce briefly the theory of ordinary kriging in this section.

Let $Z(x)$ be a random field and $z(x)$ be its realization at a spatial location $x$. We wish to estimate an unknown value $z\left(x_{0}\right)$ using observed data $\left\{z\left(x_{i}\right), i=1,2, \ldots, m\right\}$ and combine them linearly with weights $\lambda_{i 0}$ :

$$
z^{*}\left(x_{0}\right)=\sum_{i=1}^{m} \lambda_{i 0} z\left(x_{i}\right) \text {. }
$$

Here $z^{*}\left(x_{0}\right)$ represents the ordinary kriging estimate of $z\left(x_{0}\right)$. The ordinary kriging assumes the following secondorder stationary properties [Eqs. (11a)., (11b), and (11c)] for the random field $\{Z(x), x \in \Omega\}$ :

$$
\begin{aligned}
E[Z(x)] & =\mu_{Z}, \\
\operatorname{Var}[Z(x)] & =\sigma_{Z}^{2}, \\
\operatorname{Cov}\left[Z\left(x_{i}\right), Z\left(x_{j}\right)\right] & =\operatorname{Cov}\left(\left|x_{i}-x_{j}\right|\right),
\end{aligned}
$$

where $\Omega$ represents the spatial extent of the study area. We require the kriging estimator to be unbiased and have minimum variance of estimation errors, that is [Eqs. (12a) and $(12 b)]$

$$
\begin{aligned}
E\left[Z^{*}\left(x_{0}\right)\right]= & E\left[Z\left(x_{0}\right)\right], \\
\text { minimizing } & \operatorname{Var}\left[Z^{*}\left(x_{0}\right)-Z\left(x_{0}\right)\right] .
\end{aligned}
$$

The unbiasedness condition [Eq. (12a)] is warranted with unit-sum weights [Eq. (13)]

$$
\sum_{i=1}^{m} \lambda_{i 0}=1
$$

Our objective is to find a set of weights $\lambda_{i 0}$ that satisfies 
the two above constraints simultaneously. We achieve this by introducing a Lagrange multiplier $\ell$ and minimizing Eq. (14):

$$
\mathrm{M} \equiv \operatorname{Var}\left[z^{*}\left(x_{0}\right)-z\left(x_{0}\right)\right]-2 \ell\left(\sum_{i=1}^{m} \lambda_{i 0}-1\right) .
$$

By substituting $\sum_{i=1}^{m} \lambda_{i} z\left(x_{i}\right)$ for $z^{*}\left(x_{0}\right)$ and differentiating $M$ with respect to $\lambda_{i 0}$ 's and $\ell$, we obtain the ordinary kriging system (Journel and Huijbregts, 1978)

$$
\begin{gathered}
\sum_{j=1}^{m} \lambda_{i 0} \gamma_{i j}+\ell=\gamma_{i 0}, \quad i=1,2, \ldots, m, \\
\sum_{i=1}^{m} \lambda_{i 0}=1,
\end{gathered}
$$

where $\gamma_{i j}=\gamma\left(\left|x_{i}-x_{j}\right|\right)$ is the semivariogram, or simply the variogram, of the random field $Z(x)$ and is defined in Eq. (16):

$$
\gamma\left(\left|x_{i}-x_{j}\right|\right)=1 / 2 E\left\{\left[Z\left(x_{i}\right)-Z\left(x_{j}\right)\right]^{2}\right\} .
$$

Equation (15) can also be expressed in matrix form

$$
\left[\begin{array}{cccccc}
\gamma_{11} & \ldots & \gamma_{1 i} & \ldots & \gamma_{1 m} & 1 \\
\vdots & \vdots & \vdots & & \vdots & \vdots \\
\gamma_{i 1} & \ldots & \gamma_{i 1} & \ldots & \gamma_{i m} & 1 \\
\vdots & & \vdots & \vdots & \vdots & \vdots \\
\gamma_{m 1} & \ldots & \gamma_{m i} & \cdots & \gamma_{m m} & 1 \\
1 & \ldots & 1 & \ldots & 1 & 0
\end{array}\right] \cdot\left[\begin{array}{c}
\lambda_{10} \\
\vdots \\
\lambda_{i 0} \\
\vdots \\
\lambda_{m 0} \\
\ell
\end{array}\right]=\left[\begin{array}{c}
\gamma_{10} \\
\vdots \\
\gamma_{i 0} \\
\vdots \\
\gamma_{m 0} \\
1
\end{array}\right] .
$$

We solve the ordinary kriging system for kriging weights $\lambda_{i 0}$ and then substitute them into Eq. (10) to obtain $\hat{z}\left(x_{0}\right)$, the kriging estimate of $z\left(x_{0}\right)$. The variance of the estimation error, also known as the ordinary kriging variance, is given in Eq. (18) (Journel and Huijbregts, 1978):

$$
\begin{aligned}
\sigma_{o k}^{2} & =E\left\{\left[Z\left(x_{0}\right)-\hat{Z}\left(x_{0}\right)\right]^{2}\right\} \\
& =\ell+\sum_{i=1}^{m} \lambda_{i 0} \gamma_{i 0} .
\end{aligned}
$$

\begin{tabular}{|c|c|c|c|c|}
\hline Model Type & Formula & & $\begin{array}{l}\text { Influence } \\
\text { Range }\end{array}$ & Sill \\
\hline Exponential & $\gamma(h)=\omega[1-\exp (-h / a)]$ & & $3 a$ & $\omega$ \\
\hline Spherical & $\gamma(h)=\left\{\begin{array}{r}\omega\left[3 / 2(h / a)-1 / 2(h / a)^{3}\right], \\
\omega,\end{array}\right.$ & $\begin{array}{l}h \leqslant a \\
h>a\end{array}$ & $a$ & $\omega$ \\
\hline Gaussian & $\gamma(h)=\omega\left[1-\exp \left[-(h / a)^{2}\right]\right]$ & & $\sqrt{3} a$ & $\omega$ \\
\hline Power & $\gamma(h)=\omega h^{a}, a<2$ & & $+\infty$ & N/A \\
\hline
\end{tabular}

The variogram represents spatial variation structure of the random field $Z(x)$. Figure 1 illustrates the typical variogram of a random field. A variogram has three param-
Table 1. Variogram Models

eters: the influence range $h_{c}$, the sill $\gamma(\infty)$, and the nugget $C_{0}$. The influence range is the minimum distance at which two random variables $Z(x)$ and $Z\left(x+h_{c}\right)$ become independent. The sill is the lowest upper bound of the variogram and is equal to $\sigma_{Z}^{2}$, the variance of $Z(x)$. The nugget is used to model a discontinuity at the origin of the variogram. The variogram can be estimated using observed data $\left\{z\left(x_{i}\right), i=1,2, \ldots, m\right\}$

$$
\hat{\gamma}(h)=\frac{1}{2|N(h)|} \sum_{N(h)}\left[z\left(x_{i}\right)-z\left(x_{j}\right)\right]^{2}, \quad h>0
$$

where $N(h) \equiv\left\{\left(x_{i}, x_{j}\right):\left|x_{i}-x_{j}\right|=h ; i, j=1,2, \ldots, m\right\}$. The function $\hat{\gamma}(h)$ is often termed the experimental variogram. Variograms are conditionally negative definite functions (Wackernagel, 1995) and permissible models (see Table 1) or their linear combinations are often used to fit experimental variograms.

In some cases the spatial variation structures or the variograms may vary in different directions; then the random field $Z(x)$ is said to be anisotropic. For example, physical or environmental parameters such as rainfall and elevation may exhibit higher degree of variation in one direction than in the other direction. There are two types of anisotropy, the geometric anisotropy and the zonal anisotropy. The geometric anisotropy is characterized by varying influence ranges and constant sill in different directions. For zonal anisotropy, both the influence range and sill vary with directions. In dealing with the geomet-
Figure 1. Typical variogram of a random field.

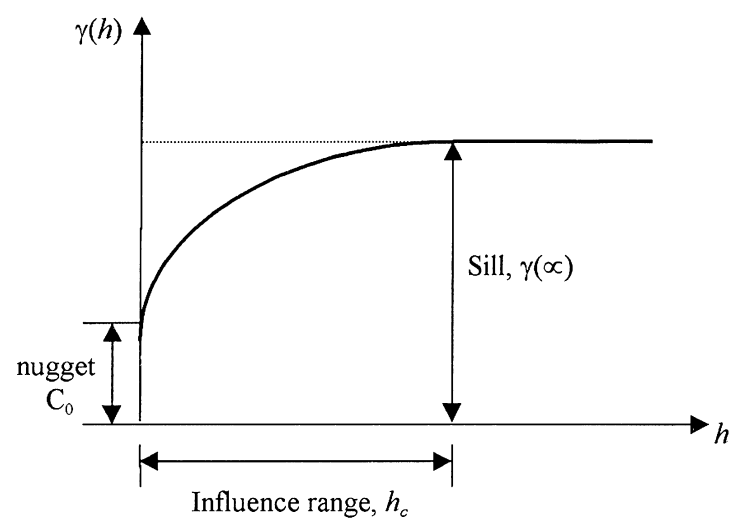

Figure 2. Rose diagram of an anisotropic random field.

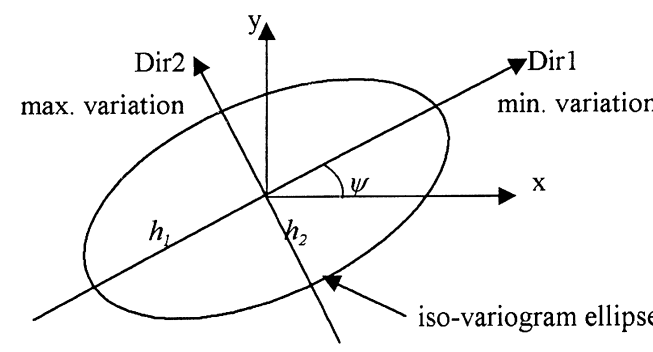

$\Psi$ : Rotation angle, anisotropic ratio, $k=h_{1} / h_{2}$ 
rically anisotropic random field, we first identify the directions of maximum and minimum spatial variation by constructing the rose diagram, an isovariogram ellipse as a function of influence ranges (see Fig. 2). We then rescale the distance between two spatial points $\left(x_{1}, y_{1}\right)$ and $\left(x_{2}, y_{2}\right)$ by using the rotation angle $\psi$ and the anisotropic ratio $k$ [Eq. (20)]:

$$
h=\sqrt{\frac{\left[\left(x_{1}-x_{2}\right) \cos \psi+\left(y_{1}-y_{2}\right) \sin \psi\right]^{2}}{+k^{2}\left[\left(y_{1}-y_{2}\right) \cos \psi-\left(x_{1}-x_{2}\right) \sin \psi\right]^{2}}}
$$

Then the spatial variation between any pair of $\left(x_{1}, y_{1}\right)$ and $\left(x_{2}, y_{2}\right)$ is calculated by using the above $h$ in the variogram model of the minimum variation axis.

\section{KRIGING APPROACH OF IMAGE RECTIFICATION}

One may consider image rectification as the work of mapping image coordinates to map coordinates through a transformation function. In terms of kriging, it seems intuitively reasonable to define a random field $Z(x, y)$ with $(x, y)$ being the image coordinate and $Z(x, y)$ the east (or north) map coordinate, and then estimate the map coordinate $Z\left(x_{0}, y_{0}\right)$ of a non-GCP pixel at $\left(x_{0}, y_{0}\right)$ using ground control points $\left\{\left(x_{i}, y_{i}\right), i=1,2, \ldots, m\right\}$ and Eqs. (10) and (17). However, since $(x, y)$ and $Z(x, y)$, respectively, represent the image coordinate and map coordinate of the same pixel, the random field $Z(x, y)$ is nonstationary, and therefore violates the ordinary kriging's second-order stationarity assumption. This modeling approach may encounter problems of fitting the experimental variograms with impermissible models, and we demonstrate this with an example in the Model Application section. In order to apply ordinary kriging to image rectification, we first model the trend present in the map-coordinate random field $Z(x, y)$ by first-degree polynomials in Eqs. (21a) and (21b):

$$
\begin{aligned}
& \hat{X}=a_{00}+a_{10} x+a_{11} y, \\
& \hat{Y}=b_{00}+b_{10} x+b_{11} y .
\end{aligned}
$$

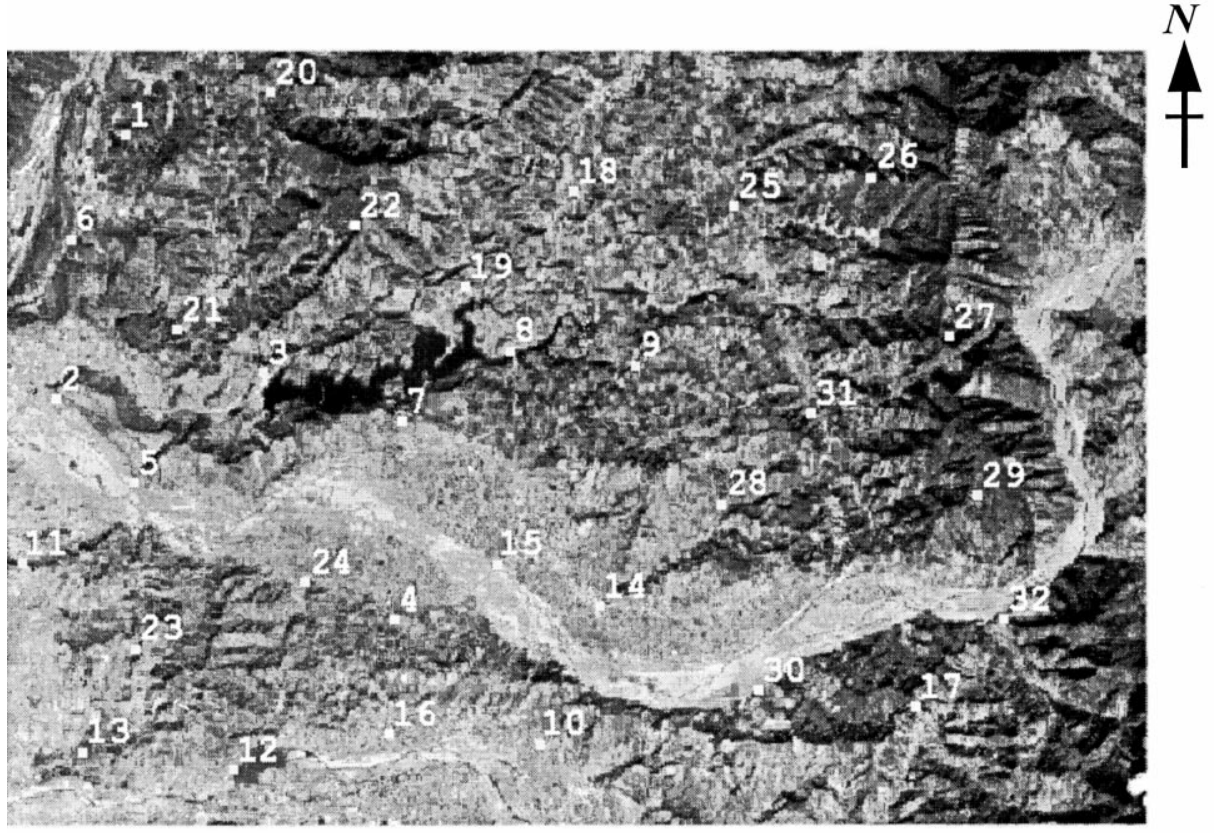

a

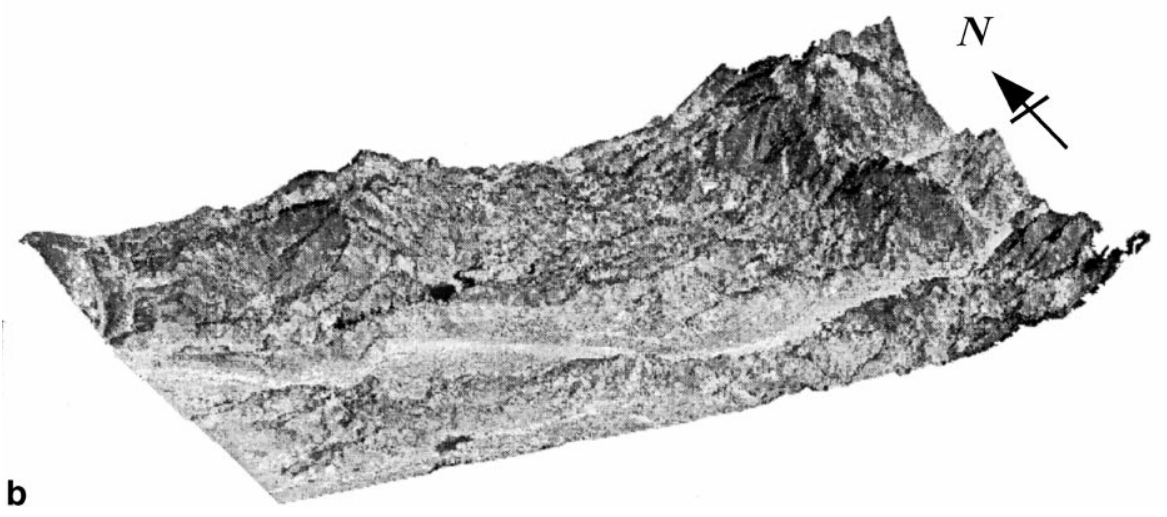

Figure 3. SPOT image of the study area. 
We then consider the residuals $d X$ and $d Y$ as two separate second-order stationary random fields, $Z_{1}(x, y)$ and $Z_{2}(x, y)$, that is,

$$
\begin{aligned}
& Z_{1}(x, y) \equiv d X=X-\hat{X}, \\
& Z_{2}(x, y) \equiv d Y=Y-\hat{Y} .
\end{aligned}
$$

In the above equation, subscripts 1 and 2 correspond to $X$ (east) and $Y$ (north) directions, respectively. The reason for using first-degree polynomials to model the trend of $Z(x, y)$ is that using higher-degree polynomials in trend mapping may eliminate the embedding spatial variation structure of the random field, and the residuals would simply be noises. Variograms of the residual random fields $Z_{1}(x, y)$ and $Z_{2}(x, y)$ must be estimated using Eq. (19) and fitted with permissible variogram models. Let $Z_{1}^{*}(x, y)$ and $Z_{2}^{*}(x, y)$ be the ordinary kriging estimates of $Z_{1}(x, y)$ and $Z_{2}(x, y)$, respectively, and they are calculated using Eqs. (10) and (17). Finally, we calculate the kriging estimate, $\left(X^{*}, Y^{*}\right)$, of map coordinate $(X, Y)$ using the following equations:

$$
\begin{aligned}
& X^{*}=\hat{X}+Z_{1}^{*}(x, y), \\
& Y^{*}=\hat{Y}+Z_{2}^{*}(x, y) .
\end{aligned}
$$

The final estimation errors of the map coordinate $(X, Y)$ by the kriging approach are

$$
\begin{aligned}
& X-X^{*}=X-\hat{X}-Z_{1}^{*}(x, y)=Z_{1}(x, y)-Z_{1}^{*}(x, y), \\
& Y-Y^{*}=Y-\hat{Y}-Z_{2}^{*}(x, y)=Z_{2}(x, y)-Z_{2}^{*}(x, y) .
\end{aligned}
$$

Equations (24a) and (24b) indicates that the final estimation errors of the kriging approach $\left(X-X^{*}, Y-Y^{*}\right)$ are numerically the same as the kriging errors $\left[Z_{1}(x, y)-\right.$ $\left.Z_{1}^{*}(x, y), Z_{2}(x, y)-Z_{2}^{*}(x, y)\right]$.

\section{ACCURACY ASSESSMENT}

Similar to the MIF technique, the ordinary kriging is also an exact interpolator that yields zero interpolation errors at data locations. In this study we apply a cross-validation technique to verify various assumptions about the ordinary kriging model and the data, and also to compare the mapping accuracies of different techniques. In the crossvalidation procedure one removes each sample value at a time from the observed data set, and reestimates the removed data from remaining samples using the different polynomial or variogram models. The method of cross validation for accuracy assessment works as follows:

1. From the GCP data set $\left\{\left(x_{i}, y_{i}\right) ;\left(X_{i}, Y_{i}\right), i=1,2\right.$, $\ldots, m\}$, choose any one point $\left[\left(x_{k}, y_{k}\right) ;\left(X_{k}, Y_{k}\right)\right]$ and use the remaining GCPs to estimate $\left(X_{k}, Y_{k}\right)$ by using the PTM, MIF, and ordinary kriging techniques.

2. Calculate the estimation errors. Let $\left(d X_{k}, d Y_{k}\right)$, $\left(d X_{M k}, d Y_{M k}\right)$, and $\left(d X_{k}^{*}, d Y_{k}^{*}\right)$ be estimation errors of the PTM, MIF, and the kriging approaches, respectively.
3. Repeat steps 1 and 2 by returning the selected GCP and choosing another point, until all ground control points have been selected.

4. Calculate and compare the means and variances of $\left(d X_{k}, d Y_{k}\right),\left(d X_{M k}, d Y_{M k}\right)$ and $\left(d X_{k}^{*}, d Y_{k}^{*}\right), k=1$, $2, \ldots, m$. A better rectification model shall have mean error close to zero and smaller variance of estimation errors.

Note that steps $1-3$ of the cross validation establish $m$ sets of polynomial and variogram models. Based on estimates of the cross validation procedure, we can also check the unbiasedness assumption of kriging [Eq. (12a)] and the coherence of kriging variance using Eqs. (25a) and $(25 \mathrm{~b})$ :

$$
\begin{gathered}
\mathrm{ME}=\frac{1}{m} \sum_{i=1}^{m}\left[z\left(x_{i}\right)-z^{*}\left(x_{i}\right)\right] \cong 0, \\
\mathrm{MRV}=\frac{1}{m} \sum_{i=1}^{m} \frac{\left[z\left(x_{i}\right)-z^{*}\left(x_{i}\right)\right]^{2}}{\sigma_{o k, i}^{2}} \cong 1,
\end{gathered}
$$

where $\sigma_{o k, i}^{2}$ is the ordinary kriging variance at location $x_{i}$.

Figure 4. Variogram parameter $a$ of $Z_{1}(x, y)$ and $Z_{2}(x$, $y$ ) in different directions (influence range $=3 a$ ).
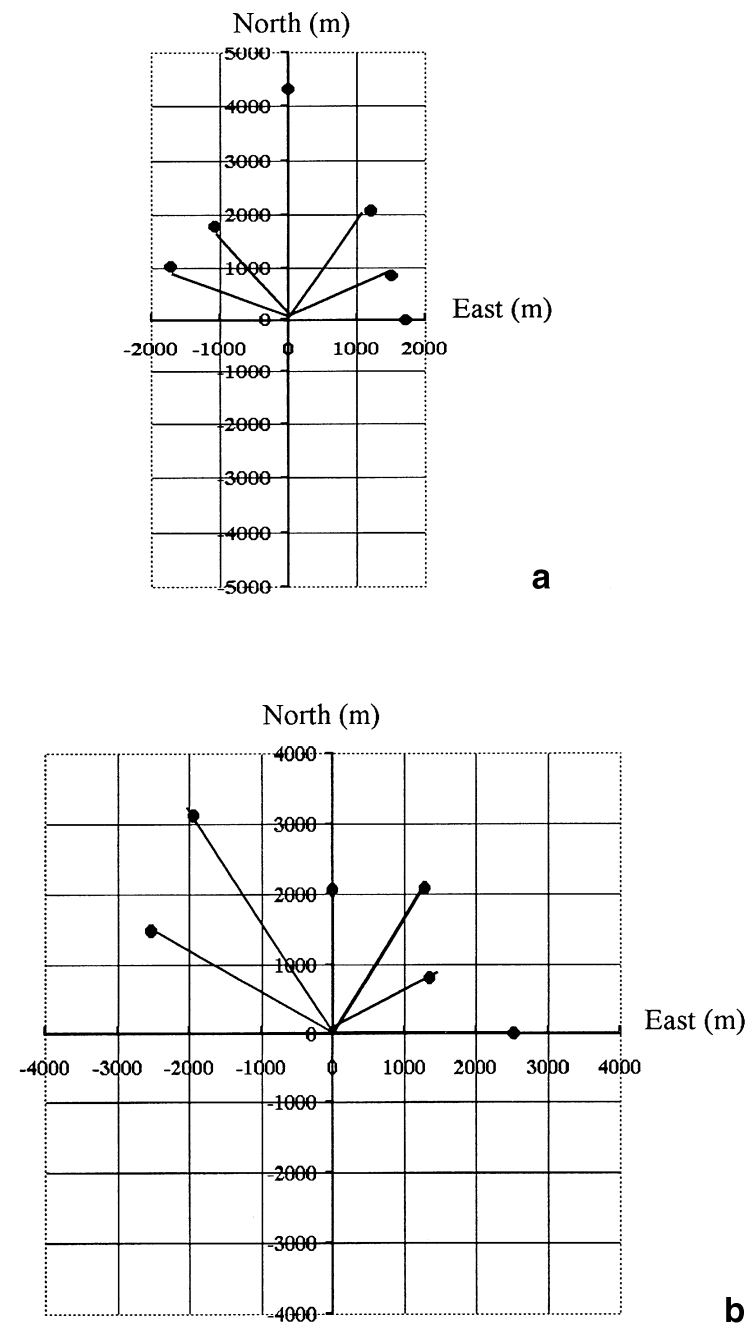

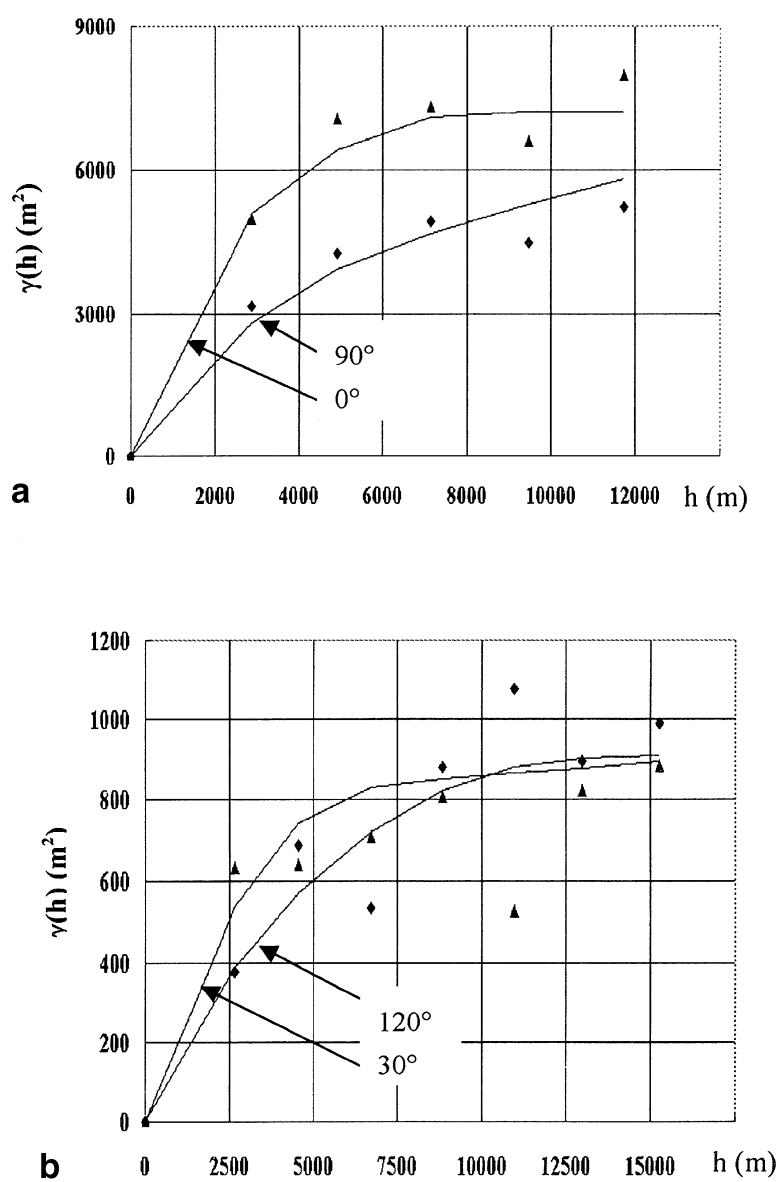

Figure 5. Major and minor directional variograms of $Z_{1}(x$, $y)$ and $Z_{2}(x, y)$.

\section{MODEL APPLICATION}

We apply the proposed ordinary kriging approach to a test area in Northern Taiwan. The test area is $20 \mathrm{~km}$ by $14 \mathrm{~km}$ in coverage, with terrain elevation ranging from $150 \mathrm{~m}$ to $1000 \mathrm{~m}$ above the mean sea level. A river flows from east to west crossing the center of the study area. We used an unrectified SPOT satellite image (see Fig. 3) covering the study area to evaluate different rectification techniques. A total of 32 ground control points were selected for model building. In the application we use the Universal Transverse Mercator (UTM) coordinates and image lines and columns for map and image coordinates, respectively.

\section{Polynomial Trend}

Using the selected GCPs, the first-degree polynomial trend present in the study area is given in Eq. (26):

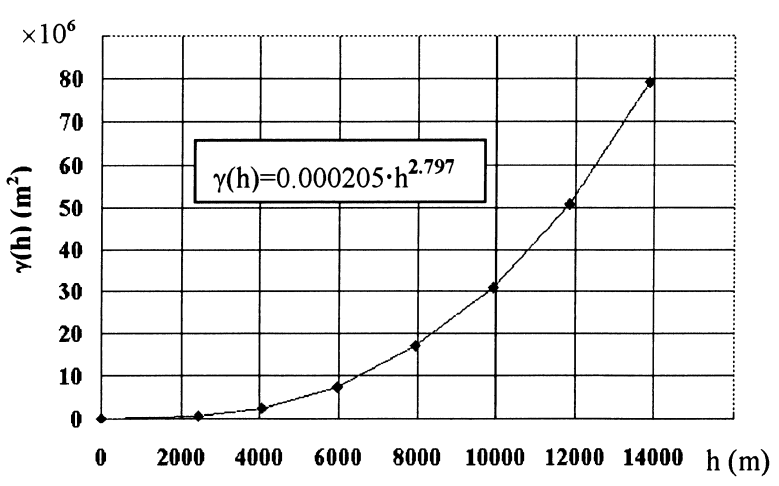

Figure 6. Power variogram of the east map ordinate.

$\left[\begin{array}{l}\hat{X}_{k} \\ \hat{Y}_{k}\end{array}\right]=\left[\begin{array}{rr}20.1298 & -2.8702 \\ -2.8702 & -19.6934\end{array}\right] \cdot\left[\begin{array}{l}x_{k} \\ y_{k}\end{array}\right]+\left[\begin{array}{r}223499.217 \\ 2699844.881\end{array}\right]$.

We then removed the trend from $Z(x, y)$ using Eqs. (22a) and (22b), and performed variogram analyses on residual random fields $Z_{1}(x, y)$ and $Z_{2}(x, y)$.

\section{Variogram Modeling}

Experimental directional variograms of residual random fields $Z_{1}(x, y)$ and $Z_{2}(x, y)$ were fitted with exponential models. The principal directions of anisotropy are $0^{\circ}-$ $180^{\circ}(\mathrm{E}-\mathrm{W})$ and $90^{\circ}-270^{\circ}(\mathrm{N}-\mathrm{S})$ for $Z_{1}(x, y)$, and $30^{\circ}$ $210^{\circ}$ and $120^{\circ}-300^{\circ}$ for $Z_{2}(x, y)$, as demonstrated in Figure 4 . The anisotropic ratios are 2.51 and 2.33 in the east and north directions, respectively. Within the study area, terrain variation is most significant in the $\mathrm{E}-\mathrm{W}$ direction and mild in the $\mathrm{N}-\mathrm{S}$ direction, therefore, more severe geometric distortions can be expected in the E-W direction. The fact that the principal directions of anisotropy for $Z_{1}(x, y)$ and $Z_{2}(x, y)$ are consistent with the principal variation directions of terrain elevation demonstrates the advantage of anisotropic variogram modeling. Figure 5 illustrates the major and minor variograms of $Z_{1}(x, y)$ and $Z_{2}(x, y)$. Also, we mentioned in earlier section that constructing variograms of the map coordinates $X$ and $Y$ without removing their trends may lead to fitting the experimental variograms with impermissible models. Figure 6 shows the experimental variogram of the east ordinate $X$, along with its best-fit power model. Since the trend present in the east ordinates is not removed, the best-fit power model has a degree of power exceeding 2.0 , and therefore such variogram model is not permissible.

Table 2. Cross Validation of Kriging Estimates

\begin{tabular}{|c|c|c|c|}
\hline Random Field & $\begin{array}{l}\text { Mean Error }(m) \\
\text { (Unbiasedness) }\end{array}$ & $\begin{array}{c}\text { Mean of Variance Ratio } \\
\text { (Variance Coherence) }\end{array}$ & Anisotropic Ratio $k$ \\
\hline$Z_{1}$ (Easting) & -0.22 & 1.06 & 2.52 \\
\hline$Z_{2}$ (Northing) & -0.15 & 1.02 & 2.33 \\
\hline
\end{tabular}


Table 3. Statistical Properties of Rectification Errors

\begin{tabular}{|c|c|c|c|c|c|c|c|c|}
\hline \multicolumn{9}{|c|}{ Rectification Errors } \\
\hline \multirow[b]{2}{*}{ Parameter } & \multicolumn{2}{|c|}{$\begin{array}{c}\text { PTM } \\
\text { (1st Degree } \\
\text { Polynomials) }\end{array}$} & \multicolumn{2}{|c|}{$\begin{array}{c}\text { PTM } \\
\text { (2nd Degree } \\
\text { Polynomials) }\end{array}$} & \multicolumn{2}{|c|}{$\begin{array}{l}\text { Multiquadric } \\
\text { Interpolation } \\
\text { Function }\end{array}$} & \multicolumn{2}{|c|}{ Ordinary Kriging } \\
\hline & $d X_{k}$ & $d Y_{k}$ & $d X_{k}$ & $d Y_{k}$ & $d X_{M k}$ & $d Y_{M k}$ & $d X^{*}$ & $d Y^{*}$ \\
\hline Mean (m) & 1.37 & -0.28 & -0.18 & 0.15 & 0.22 & -0.75 & -0.22 & -0.15 \\
\hline Variance $\left(\mathrm{m}^{2}\right)$ & 7978.59 & 876.30 & 7609.78 & 553.95 & 7368.94 & 642.27 & 4803.02 & 581.05 \\
\hline \multirow[t]{2}{*}{$\operatorname{RMSE}(\mathrm{m})$} & 87.93 & 29.14 & 85.86 & 23.19 & 84.49 & 24.96 & 68.21 & 23.73 \\
\hline & \multicolumn{2}{|c|}{$65.50^{a}$} & \multicolumn{2}{|c|}{$62.89^{a}$} & \multicolumn{2}{|c|}{$62.30^{a}$} & \multicolumn{2}{|c|}{$51.07^{a}$} \\
\hline \multicolumn{9}{|c|}{ Absolute Rectification Errors } \\
\hline & \multicolumn{2}{|c|}{$\begin{array}{c}\text { PTM } \\
\text { (1st Degree } \\
\text { Polynomials) }\end{array}$} & \multicolumn{2}{|c|}{$\begin{array}{c}\text { PTM } \\
\text { (2nd Degree } \\
\text { Polynomials) }\end{array}$} & \multicolumn{2}{|c|}{$\begin{array}{c}\text { Multiquadric } \\
\text { Interpolation } \\
\text { Function }\end{array}$} & \multicolumn{2}{|c|}{ Ordinary Kriging } \\
\hline Parameter & $\left|d X_{k}\right|$ & $\left|d Y_{k}\right|$ & $\left|d X_{k}\right|$ & $\left|d Y_{k}\right|$ & $\left|d X_{M k}\right|$ & $\left|d Y_{M k}\right|$ & $\left|d X^{* *}\right|$ & $\left|d Y^{*}\right|$ \\
\hline Mean $(\mathrm{m})$ & 68.26 & 23.09 & 63.69 & 18.57 & 59.56 & 20.62 & 52.57 & 19.00 \\
\hline Variance $\left(\mathrm{m}^{2}\right)$ & 3171.13 & 326.06 & 3421.97 & 197.98 & 3706.87 & 203.94 & 1950.43 & 208.39 \\
\hline
\end{tabular}

${ }^{a}$ Overall RMSE

\section{Accuracy Assessment}

The final estimates of map coordinates for all GCPs were calculated using the anisotropic variogram models and Equations (10), (17), (23a), and (23b). Table 2 presents the results of cross-validation check of kriging estimates. The unbiasedness of kriging estimates $(\mathrm{ME} \simeq 0)$ and coherence of kriging variance $(\mathrm{MRV} \simeq 1)$ are well satisfied, indicating the anisotropic variogram models and the assumption of second-order stationarity for the residual random fields $Z_{1}(x, y)$ and $Z_{2}(x, y)$ (after variogram rescaling using the anisotropic ratio) are adequate for this application. Table 3 presents the statistical properties of rectification errors $\left(d X_{k}, d Y_{k}\right),\left(d X_{M k}, d Y_{M k}\right)$, and $\left(d X_{k}^{*}\right.$, $\left.d Y_{k}^{*}\right)$ based on the cross-validation procedures outlined in previous section. The ordinary kriging approach yields smaller mean, variance and root-mean-square of estimation errors than the MIF and PTM with the first-degree polynomials. Although PTM with the second-degree polynomials achieves slightly smaller mean and variance of estimation errors in the north direction than the kriging approach, their differences are negligible. More importantly, the kriging approach achieves approximately

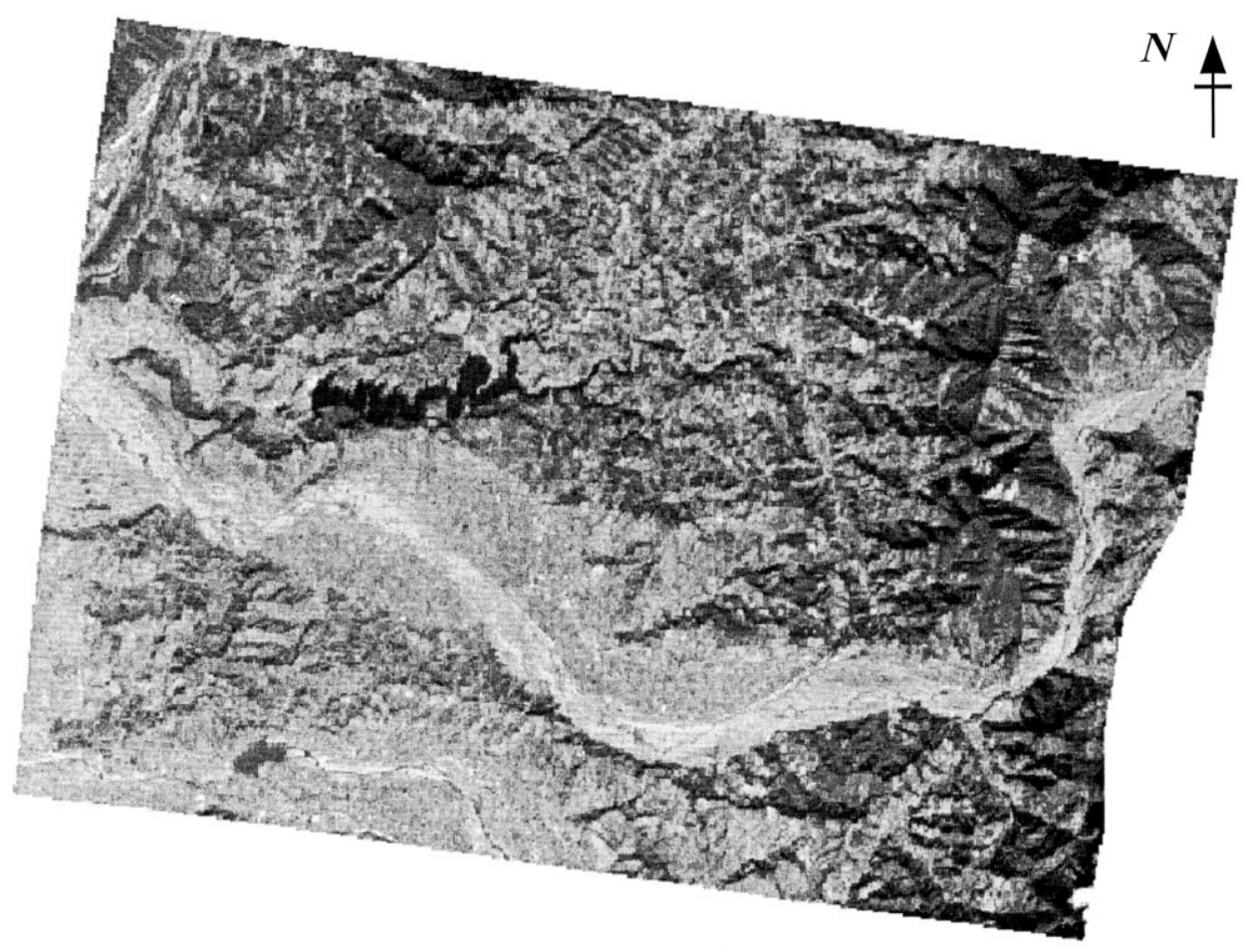

Figure 7. Kriging rectified image. 
$35-40 \%$ reductions in variance of estimation errors in the east direction than all other models. This improvement is admirable since the terrain variation and geometric distortions are most significant in the east direction. Also, root-mean-square error (RMSE) of horizontal (east) direction is significantly higher than RMSE of vertical (north) direction, indicating the rectification errors are mainly contributed by errors in the east direction. The overall RMSE of the ordinary kriging is $51.07 \mathrm{~m}, 18-22 \%$ lower than RMSE of other models. Finally the kriging rectified image of the study area is shown in Figure 7.

\section{CONCLUSIONS}

In this study we compare the accuracies of image rectification by polynomial trend mapping, multiquadric interpolation function, and ordinary kriging techniques. The proposed ordinary kriging approach of image rectification considers the mapping errors resulted from traditional polynomial trend mapping as two stationary random fields. Anisotropic variogram modeling of the PTM residuals identifies the principal variation directions and then take into account the spatial variation structures of different directions. Results of the cross validation demonstrate that ordinary kriging approach yields smaller values of variance and root mean square of mapping errors than PTM and MIF techniques. This study demonstrates the potential of applying the proposed ordinary kriging approach to image rectification for both space-borne and airborne images.

The first author would like to thank the National Science Council of Taiwan, R.O.C. for financially supporting his one-year visit to the Mathematics Department, University of Florida, during which this study was carried out. He is also grateful to the Mathematics Department of University of Florida for providing excellent research environment. We also thank the anonymous reviewers for their constructive criticisms and suggestions that were very helpful in revising the manuscript.

\section{REFERENCES}

Chilès, J. P., and Delfiner, P. (1999), Geostatistics-Modeling Spatial Uncertainty, Wiley, New York, pp. 449-451.

Haralick, R. M. (1973), Glossary and index to remotely sensed image pattern recognition concepts. Pattern Recognition 5: 391-403.

Hardy, R. L. (1990), Theory and applications of the multiquadric-biharmonic method. Comput. Math. Appl. 19(8/9):163-208.

Jensen, J. R. (1986), Introductory Digital Image Processing-A Remote Sensing Perspective, Prentice-Hall, Englewood Cliffs, NJ, pp. 102-105.

Journel, A. G., and Huijbregts, C. J. (1978), Mining Geostatistics, Academic, London, 600 pp.

Malinverno, A. (1988), Testing linear models of sea-floor topography. Pure Appl. Geophys. 131:139-156.

Paine, D. P. (1981), Aerial Photography and Image Interpretation for Resource Management, Wiley, New York, 571 pp.

Richards, J. A. (1995), Remote Sensing Digital Image Analysis, Springer-Verlag, Berlin, pp. 54-57.

Schowengerdt, R. A. (1997), Remote Sensing-Models and Methods for Image Processing, Academic, San Diego, pp. 324-353.

Star, J. L., Estes, J. E., and McGwire, K. C. (1997), Integration of Geographic Information Systems and Remote Sensing, Cambridge University Press, New York, pp. 18-25.

Turcotte, D. L. (1998), Fractals in geology and geophysics. Pure Appl. Geophys. 131:171-196.

Wackernagel, H. (1995), Multivariate Geostatistics, Springer-Verlag, Berlin, pp. 37-38. 